\title{
Does childhood trauma play a role in the aetiology of psychosis? A review of recent evidence ${ }^{\dagger}$
}

\author{
Helen Coughlan \& Mary Cannon
}

\begin{abstract}
SUMMARY
There has been a resurgence of interest in the role of childhood trauma in the aetiology of psychosis. In this review, recent findings on the association between childhood trauma and a continuum of psychotic symptoms are presented. Evidence of the association between specific childhood trauma subtypes and psychotic symptoms is examined, with a brief discussion of some current hypotheses about the potential mechanisms underlying the associations that have been found. Some practice implications of these findings are also highlighted.
\end{abstract}

\section{LEARNING OBJECTIVES}

- Identify findings from recent meta-analyses on the association between childhood trauma and a range of psychotic outcomes, from non-clinical psychotic experiences to psychotic disorders

- Consider which childhood traumas are the most potent in the context of psychotic outcomes

- Recognise that the relationships between childhood trauma, psychotic symptoms and other psychopathology are complex, dynamic and multidimensional

\section{DECLARATION OF INTEREST}

None

'Stress or trauma of some kind is, I believe [...] a factor in every case of psychosis, and every human being, has, I think, a threshold of vulnerability' (MacDougall 1939).

A key imperative in current psychosis research is the development of an integrated and nuanced understanding of how and why psychotic symptoms emerge and, for a small minority, become entrenched and result in significant social and functional impairment (i.e. disorder). A dynamic range of interrelated factors have been implicated in the activation and perpetuation of psychosis, among which there is growing interest in the role of early childhood trauma. However, with low rates of conversion to psychotic disorder, even in at-risk groups, questions still remain about the level of risk conferred by exposure to childhood trauma and the range of potential mechanisms through which such exposure is converted into psychotic symptoms or disorders.

This article is based on our review of reported findings on the relationship between childhood trauma and psychosis. For it, we reviewed papers from the time of publication of a large meta-analysis by Varese and colleagues in 2012 to the time our article was submitted in November 2016. We did not follow the process of a systematic review, so some relevant studies may not be included. Table 1 summarises the key studies that we identified.

\section{The association between childhood trauma and psychotic outcomes}

The idea that trauma and stress are implicated in the emergence and persistence of psychotic symptoms and disorders is not new (e.g. Clark 1932; MacDougall 1939), and evidence from a number of recent studies has confirmed that childhood trauma and adversity are associated with a continuum of outcomes along the psychosis spectrum, from non-clinical psychotic experiences to diagnosable psychotic disorders (Arseneault 2011; Bentall 2012; Varese 2012; Dvir 2013; Fisher 2013; Kelleher 2013a; Matheson 2013; Sheffield 2013; Bentall 2014; Duhig 2015; Kraan 2015; Trotta 2015; Ajnakina 2016).

In a meta-analysis, Varese and colleagues (2012) examined data on childhood trauma and psychosis from 41 population-based, case-control and prospective studies. For the analysis, they defined childhood trauma as any history of sexual, physical or emotional abuse, neglect, parental death or bullying, and outcomes included both diagnostic (i.e. any diagnosed psychotic disorder) and dimensional (i.e. non-clinical hallucinations and delusions) measures of psychosis. The authors found an almost 3-fold (OR 2.79, 95\% CI 2.343.31) increase in the odds of psychotic outcomes among people exposed to childhood trauma. This increased risk was evident across both populationbased and clinical case-control studies.

\section{ARTICLE}

Helen Coughlan is a Clinical Research Fellow in the Department of Psychiatry at the Education and Research Centre, Beaumont Hospital, Dublin. A social worker by profession, she has worked across the public, private and non-governmental organisation (NGO) mental health sectors. Awarded a Health Research Board Health Professionals Fellowship in 2015, Helen is currently conducting a PhD study on the relationship between adverse life experiences and hallucinations and delusions in young people. Mary Cannon is Professor of Psychiatric Epidemiology at the Royal College of Surgeons in Ireland and a consultant liaison psychiatrist in the Department of Psychiatry at Beaumont Hospital, Dublin. Her research is focused on risk factors and neurobiological markers for psychotic symptoms in youth populations.

Correspondence Helen Coughlan, Royal College of Surgeons in Ireland, RCSI Education and Research Centre, Beaumont Hospital, Dublin 9, Ireland. Email: helencoughlan@ rcsi.ie

\section{Copyright and usage}

(C) The Royal College of Psychiatrists 2017

\footnotetext{
${ }^{\dagger}$ For commentaries on this article, see pp. 316-317 and 318-320, this issue.
} 
Meta-analyses on childhood trauma/adversity and psychotic outcomes, 2012-2015

\begin{tabular}{|c|c|c|c|c|c|}
\hline Authors & $\begin{array}{l}\text { Studies included, } \\
n\end{array}$ & $\begin{array}{l}\text { Sample size } \\
\text { range, } n\end{array}$ & $\begin{array}{l}\text { Age range, } \\
\text { years }\end{array}$ & $\begin{array}{l}\text { Type/s of childhood } \\
\text { trauma examined }\end{array}$ & Main findings \\
\hline Varese et al (2012) & 41 & $35-17337$ & $12-78$ & $\begin{array}{l}\text { Sexual abuse, physical abuse, } \\
\text { emotional/psychological abuse, } \\
\text { neglect, parental death, bullying }\end{array}$ & $\begin{array}{l}\text { Childhood trauma was associated } \\
\text { with psychotic outcomes in } \\
\text { population-based, clinical and } \\
\text { prospective study samples }\end{array}$ \\
\hline van Dam et al (2012) & 14 & $64-8580$ & $12-74$ & Bullying & $\begin{array}{l}\text { Bullying was associated with } \\
\text { psychotic outcomes among } \\
\text { population-based study samples. } \\
\text { Findings were inconclusive for } \\
\text { clinical/patient populations }\end{array}$ \\
\hline Vassos et al (2012) & 4 & $26-17389$ & $18+$ & 'Urbanicity' (urban living) & $\begin{array}{l}\text { Childhood urban living was } \\
\text { associated with psychotic outcomes }\end{array}$ \\
\hline Bonoldi et al (2013) & 23 & $18-569$ & $16-65$ & $\begin{array}{l}\text { Sexual abuse, physical abuse, } \\
\text { emotional abuse }\end{array}$ & $\begin{array}{l}\text { Higher prevalence of childhood } \\
\text { sexual, physical and emotional } \\
\text { abuse among clinical populations } \\
\text { compared with healthy controls }\end{array}$ \\
\hline Trotta et al(2015) & $\begin{array}{l}20 \text { reviewed; } \\
9 \text { included }\end{array}$ & $71-9292$ & Not reported & $\begin{array}{l}\text { Sexual abuse, physical abuse, } \\
\text { emotional/psychological abuse, } \\
\text { neglect, parental death, bullying, } \\
\text { life-threatening accident, natural } \\
\text { disaster, victim of violence, parental } \\
\text { conflict, paternal mental disorder, } \\
\text { punitive parenting, direct combat } \\
\text { experience in wara }\end{array}$ & $\begin{array}{l}\text { Childhood trauma was associated } \\
\text { with psychotic outcomes in both } \\
\text { population-based and clinical } \\
\text { samples }\end{array}$ \\
\hline
\end{tabular}

a. Meta-analysis also included adult traumas.

A subsequent meta-analysis by Matheson and colleagues (2013) focused specifically on patients with schizophrenia. Its authors found that schizophrenia patients were 3.6 times more likely to have experienced childhood trauma when compared with healthy controls (OR 3.60, 95\% CI 2.086.23). However, when they extended their analyses to include associations between childhood trauma and other psychopathological outcomes, the authors found that, although patients with schizophrenia were more likely to have experienced childhood trauma than patients with anxiety, there were no significant differences in rates of childhood trauma between schizophrenia patients and patients with affective psychoses, depression or personality disorders. This finding reflects the real challenge in determining exact pathways that lead from certain childhood traumas to specific psychopathological outcomes, including psychosis.

Most recently, a meta-analysis by Trotta and colleagues (2015) found that exposure to childhood trauma conferred an almost 1.8-fold increase in the odds of hallucination and delusion persistence among general population samples (OR 1.76, 95\% CI 1.19-2.32), with an over 1.5-fold increase in the odds of psychotic symptom persistence among individuals already diagnosed with a psychotic disorder (OR 1.55, 95\% CI 0.32-2.77). Although these findings in the studies examined point to the potentially enduring impact that childhood trauma may have on both the emergence and the later evolution of psychotic symptoms, it is important to note that only nine papers met inclusion criteria for the meta-analysis: five of these were general population studies and four involved clinical samples. In addition, the authors urge that the findings be considered cautiously because a number of methodological issues may have affected the reliability of some of the findings of association across the studies.

In addition to theindividual risk that accompanies the experience of a range of childhood traumas, evidence also supports the hypothesis that multiple or cumulative traumas provide an additive risk effect on psychotic outcomes in both populationbased (Shevlin 2011a; Bentall 2012; Kelleher 2013a) and clinical samples (Muenzenmaier 2015; Longden 2016). This suggests that individuals who experience multiple traumas are at higher risk of both non-clinical and clinical psychotic outcomes. However, a notable limitation in findings that have addressed the impact of multiple traumas on psychotic outcomes is that most involve only a crude count of trauma types and therefore fail to incorporate any consideration of the subjective impact of these experiences.

\section{Psychotic symptoms and trauma types}

\section{Childhood sexual abuse}

Of all childhood traumas, childhood sexual abuse has emerged as one of the most potent 
in the context of psychotic outcomes. With some exceptions (e.g. Longden 2016), a growing number of studies have demonstrated that childhood sexual abuse is strongly associated with both non-clinical psychotic experiences and psychotic disorders (Bebbington 2011; Bentall 2012; Daalman 2012; Sheffield 2013; Velthorst 2013; Morgan 2014; Thompson 2014; Ajnakina 2016). Evidence that childhood sexual abuse confers specific risk for the experience of both auditory (Shevlin 2011a; Bentall 2012; Daalman 2012; Sheffield 2013) and visual hallucinations (Shevlin 2011a) is most convincing. In one large population-based survey in the UK, people who had been subjected to rape during childhood were found to be almost 9 times more likely to experience hallucinations than those who had not (OR 8.9, 95\% CI 1.86-42.44) (Bentall 2012). Conversely, evidence that childhood sexual abuse is a risk factor for paranoia and delusions is equivocal (Bentall 2012; Sitko 2014). However, fewer studies have reported on delusional or paranoid outcomes. In addition, among ultrahigh-risk samples, childhood sexual abuse is the only trauma that has been found to be associated with transition to psychotic disorder (Bechdolf 2010; Thompson 2014). This finding suggests that childhood sexual abuse may confer specific risk for psychotic symptom persistence and the development of a pathological psychotic disorder.

\section{Childhood physical abuse}

Like childhood sexual abuse, physical abuse in childhood has been found to be associated with psychotic symptoms in both population-based (Shevlin 2011a; Bentall 2012; Fisher 2012; Beards 2013) and clinical studies (Daalman 2012; Sheffield 2013; Ajnakina 2016; Longden 2016). Among adult population-based samples, those with a history of childhood physical abuse have been found to be up to almost 9 times more likely to experience hallucinations when compared with healthy controls (OR 8.79 [95\% CI not reported]) (Daalman 2012). Using data from over 250 medical records in New Zealand, Longden and colleagues (2016) found that childhood physical abuse was associated with hallucinations (OR 2.11, 95\% CI 1.10-4.47), in particular command hallucinations (OR 3.35, 95\% CI 1.32-8.50), but not with delusions or paranoia. This is at variance with some previous studies in which physical abuse was found to increase the likelihood of experiencing paranoia in both population-based (OR 8.52, 95\% CI 3.55-20.43) (Bentall 2012) and ultra-high-risk (OR 3.03, 95\% CI 1.18-7.79) samples (Velthorst 2013).
In their study comparing individuals with clinical psychosis with a sample that experienced non-clinical auditory verbal hallucinations and a sample of healthy controls, Daalman and colleagues (2012) found higher rates of physical abuse in the non-clinical auditory verbal hallucinations group than in the clinical group, with higher associated odds (8.79 v. 7.44$)$ when compared with the healthy controls.

These varied findings raise some questions about the specificity of risk conferred by childhood physical abuse, in terms of both the risk of a pathological outcome and the types of psychotic symptoms experienced.

Among adults subjected to sexual and/ or physical abuse in childhood there is some qualitative evidence that the content of their hallucinations and delusions is directly related to their earlier experiences of these traumas (Reiff 2012). These qualitative findings are supported by data from a study of individuals at ultra-highrisk of psychosis, among whom sexual abuse was associated with more malevolent hallucinatory content. Those in the ultra-high-risk sample who reported childhood sexual abuse were 4.5 times (OR 4.5, 95\% CI 1.30-15.88) more likely to experience perceptual distortions with abusive content than those with no history of sexual abuse, even after adjusting for other traumas (Velthorst 2013).

\section{Childhood emotional abuse}

Although childhood emotional abuse has been identified as a risk factor for psychotic outcomes, reported findings have been both limited and inconsistent. Some studies have found early emotional abuse to be associated with hallucinations (Daalman 2012; Sheffield 2013), with one study finding that it conferred a 6.5 -fold increase in the likelihood of experiencing nonclinical auditory verbal hallucinations specifically (OR 6.51 [95\% CI not reported]) (Daalman 2012). In both clinical and population-based samples, there is also evidence that there is between a 2-fold (OR 2.20, 95\% CI 1.13-4.26) (Longden 2016) and 3-fold increase (OR 3.26, 95\% CI 1.56-6.81) (Fisher 2012) in the likelihood of experiencing paranoia among people subjected to childhood emotional abuse. Importantly, in one study where childhood emotional abuse was found to be highly correlated with both sexual and physical abuse, it was the cooccurrence of sexual abuse with emotional abuse that drove the association with hallucinations, suggesting that emotional abuse on its own may be less potent in its effect on later hallucinations (Sheffield 2013). 
${ }^{\ddagger}$ Gennaro Catone et a/ will present a more detailed discussion of bullying victimisation and psychosis in the next issue of BJPsych Advances. Ed.

\section{Childhood neglect}

As with emotional abuse, data on the impact of childhood neglect are limited. Among populationbased studies, childhood neglect has been found to have an association with psychotic experiences generally (van Nierop 2014) and with paranoia specifically (Sitko 2014). Differing findings have emerged from clinical population studies. One Dutch study found an association between childhood neglect and hallucinations among patients with a psychotic disorder (Daalman 2012), whereas a study from New Zealand found no such association with either hallucinations or any other psychotic symptom type (Longden 2016).

One of the challenges in relation to emotional abuse and neglect is that the parameters of how such experiences are conceptualised and defined vary considerably. In addition, as with other forms of childhood trauma, studies using adolescent or adult samples may be subject to retrospective recall bias among study participants. Notwithstanding these definitional and methodological issues, with such limited and variable evidence on the impact of childhood emotional abuse and neglect, additional prospective population-based research is needed to better understand the range of psychotic outcomes in both population and clinical samples.

\section{Loss through death and the experience of institutional care in childhood}

Although childhood trauma that involves intent to harm (i.e. child abuse) appears to be particularly salient in the emergence and persistence of psychosis (van Nierop 2014), childhood traumas without an explicit intent to harm have also emerged as risk factors for psychosis. There are a number of childhood traumas that involve some degree of loss and a resultant discontinuation in established family and other relationships and/ or the home environment. These include parental death and institutional care. Although death of a parent, sibling or friend was not associated with non-clinical psychotic experiences in a large population-based Dutch study (van Nierop 2014), an even larger study of over 900000 people in Sweden found that death of a close family member and, in particular, death within the nuclear family, was associated with psychotic disorders (OR 1.44, 95\% CI 1.27-1.63) (Abel 2014). The association was especially strong for deaths that occurred during the first 3 years of the child's life (OR 1.84, 95\% CI 1.41-2.41) and for deaths from suicide during that phase of the lifespan (OR 2.17, 95\% CI 1.523.08). These findings raise important questions about the mechanisms that are activated during this formative phase of development, a phase that pre-dates verbal memory. It also suggests that early losses that are traumatic for other adult caregivers in an infant's life may be implicated in the associations found.

Psychosis has also been found to be associated with the trauma of being separated from one's parent(s) and removed into institutional or foster care. In one clinical study, the experience of living in foster care was associated with verbal hallucinations, paranoia and the negative symptoms of psychosis (Longden 2016). However, in a large population-based study, institutional care was found only to be associated with paranoia, with an 11-fold increase in the odds of experiencing paranoia among individuals who had spent time in care (OR 11.08, 95\% CI 3.2637.62) (Bentall 2012).

\section{Childhood bullying}

The relationship between bullying and psychosis has been the focus of number of recent studies (Fisher 2013; Kelleher 2013a; Wolke 2014; Catone 2015; Valmaggia 2015). ${ }^{\ddagger}$ In a recent meta-analysis, bullying in childhood was found to confer a 2.3-fold increase (95\% CI 1.5-3.4) in the odds of experiencing non-clinical psychotic phenomena across population-based samples, but evidence of any association in clinical samples was inconclusive (van Dam 2012). The association between bullying and psychotic symptoms has been most evident in non-clinical adolescent samples. Data from two studies in the UK and Ireland respectively found that adolescents with a history of being bullied were between 1.5 (Fisher 2013) and 3 times (Kelleher 2013a) more likely to report nonclinical psychotic experiences than those without. In the latter study, a bidirectional association between bullying and psychotic experiences was found, highlighting a dynamic relationship between them. However, by controlling for the presence of psychotic experiences that pre-dated any experiences of bullying, the study was able to confirm an association between bullying and new-incident psychotic experiences with a doseresponse effect. Findings from this study therefore point to the possibility of a causal relationship between being bullied and the emergence of psychotic experiences in youth populations. Of particular clinical relevance was a finding by Kelleher and colleagues (2013a) that the cessation of bullying was associated with a reduction in psychotic experiences at 12-month follow-up. This important finding suggests that efforts to halt experiences of bullying could be protective against the persistence of psychotic experiences in young people. 


\section{Childhood trauma and psychosis: specific or general effects?}

The weight of recent evidence clearly implicates a range of childhood traumas in the genesis and evolution of both non-clinical and clinical psychosis trajectories. This association is evident across hallucinatory and delusional phenomena in both population-based and clinical samples (Table 2). However, findings of association do not prove causation. In addition, childhood trauma has been found to be associated with a range of other disorders, including depressive (Heim 2008; Kessler 2010), anxiety (Heim 2001) and personality disorders (Afifi 2011; Klein 2015; Velikonja 2015). Furthermore, psychotic experiences themselves are associated with a range of other non-psychotic psychopathology (Kelleher 2012; Schroeder 2013; Fusar-Poli 2014; Bortolon 2015; McCarthy-Jones 2015; Upthegrove 2015). Of particular relevance to this review is the association between psychotic symptoms and post-traumatic stress disorder (PTSD) (e.g. Mueser 2002; Kilcommons 2005; Read 2005; Shevlin 2011b). From a clinical perspective, the evidence seems to suggest that both early trauma and psychotic symptoms have transdiagnostic relevance rather than being predictive of any specific psychopathological outcome. This highlights the diagnostic and treatment challenges that accompany complex clinical presentations involving early trauma where such experiences are likely to be interacting synergistically with myriad other factors and disorders to result in psychotic outcomes.

\section{The role of biological and cognitive mechanisms}

One of the dominant current perspectives on the aetiology of psychotic disorders is that of a complex gene-environment interaction that influences both the emergence and the persistence of psychotic symptoms (van Os 2008, 2009, 2010). From this perspective, no single pathway is seen as responsible for the range of psychotic phenomena that individuals experience (Bentall 2014). Rather, it is a dynamic interplay between genetics, epigenetics, exposure to childhood trauma, and the experience of suboptimal environmental factors that causes the activation and later pathologisation of psychosis (Millan 2016). In relation to early trauma and adversity, evidence supports the view that exposure to stress, including childhood trauma, maltreatment and suboptimal environmental conditions, can affect brain (Seo 2014), biological (McEwen 2000; Hayes 2014), social (MacDonald 2000), cognitive (Kelleher 2013b; Aas 2014) and emotional development. Certain permutations of these exposures and their effects may sensitise some individuals to become more vulnerable or prone to hallucinations, delusions and psychotic disorders (van Os 2009, 2010). Along with any pre-existing genetic loading (van Os 2008), it is the experience of repeated exposure to multiple risk factors from the prenatal to the early adult years that may place individuals at highest risk for progression to pathological manifestations of psychosis (Millan 2016). Among those with exposure to stress in childhood and/or the emergence of early non-clinical psychotic experiences, additional exposure to stress during adolescence and into young adulthood could play a key role in activating the transition to pathological psychotic outcomes, with the resultant social, emotional and functional consequences that generally accompany diagnosed psychotic disorders.

\section{Psychodynamic perspectives}

From a psychodynamic perspective, the experience of psychosis has long been conceptualised as a

TABLE 2 Summary of associations found between childhood trauma and psychotic outcomes ${ }^{a}$

\begin{tabular}{|c|c|c|c|c|c|c|c|c|c|}
\hline \multirow[b]{2}{*}{ Psychosis outcome } & \multicolumn{9}{|c|}{ Childhood trauma type } \\
\hline & Any & Cumulative & $\begin{array}{l}\text { Sexual } \\
\text { abuse }\end{array}$ & $\begin{array}{l}\text { Physical } \\
\text { abuse }\end{array}$ & $\begin{array}{l}\text { Emotional } \\
\text { abuse }\end{array}$ & Neglect & $\begin{array}{c}\text { Parental } \\
\text { death }\end{array}$ & $\begin{array}{l}\text { Institutional } \\
\text { care }\end{array}$ & Bullying \\
\hline Any psychotic outcome & $\checkmark$ & $\checkmark$ & $\checkmark$ & $\checkmark$ & $\checkmark$ & $\checkmark$ & $\checkmark$ & $\checkmark$ & $\checkmark$ \\
\hline $\begin{array}{l}\text { Non-clinical psychotic } \\
\text { experiences }\end{array}$ & $\checkmark$ & $\checkmark$ & $\checkmark$ & $\checkmark$ & $\checkmark$ & $\checkmark$ & & $\checkmark$ & $\checkmark$ \\
\hline Psychotic disorder & $\checkmark$ & $\checkmark$ & $\checkmark$ & $\checkmark$ & $\checkmark$ & $\checkmark$ & $\checkmark$ & $\checkmark$ & \\
\hline Any hallucinations & & & $\checkmark$ & $\checkmark$ & $\checkmark$ & $\checkmark$ & & $\checkmark$ & \\
\hline Auditory hallucinations & & & $\checkmark$ & $\checkmark$ & $\checkmark$ & & & $\checkmark$ & \\
\hline Paranoia & & & & $\checkmark$ & $\checkmark$ & $\checkmark$ & & $\checkmark$ & \\
\hline Persistence & $\checkmark$ & & & & & & & & $\checkmark$ \\
\hline Transition to disorder & & & $\checkmark$ & & & & & & \\
\hline
\end{tabular}

a. Summary only of the studies reviewed for the current article. 
defence against unbearable or unmanageable emotions (Martindale 2013), and psychotic symptoms are understood to have meaning in the context of people's experiences (Martindale 2007). In some recent studies, for example, the content of psychotic experiences has been found to reflect specific aspects of traumatic and adverse life experiences among both high-risk and clinical populations (Reiff 2012; Velthorst 2013). Recently, the growth of the hearing voices movement and the rejection by some of the assumption that psychotic experiences are inherently pathological have resulted in the development of new theories about the mechanisms and meaning of auditory verbal hallucinations (AVHs) in particular. For example, in their extensive review on AVHs, Longden and colleagues propose that AVHs may be understood not necessarily as symptoms of a psychotic disorder, but as unconscious dissociative

\section{The CAP criteria}

Consider

- When assessing patients who present with psychotic symptoms, consider childhood trauma as a potentially significant factor in the onset and nature of their symptoms. Conversely, when assessing patients who report a history of childhood trauma, consider the possibility that they may be experiencing primary or secondary psychotic symptomatology

- Admission to a psychiatric hospital (particularly an involuntary admission) is traumatic for many people. For psychosis patients with a history of childhood trauma this has the potential to contribute additively to the impact of their earlier traumatic experiences. It is therefore always important to attend not only to the immediate presenting symptoms that resulted in the admission, but also to the potential impact on the patient of the admission process.

Ask and act

- Unless they are a part of a patient's primary presenting symptoms, people who experience psychotic symptoms often do not report them unless explicitly asked about them. For patients who report a history of childhood trauma, ask about any psychotic symptoms that they may have experienced or are currently experiencing. For patients who present with psychotic symptoms, ensure that you ask them about any history of childhood trauma.

- Take action to respond to reported experiences of childhood trauma. For patients who report a history of childhood trauma, allow space for them to talk about these experiences if they wish to do so. Discuss the potential routes that can be pursued in relation to these experiences and ask them what they would like to do in relation to their experiences. Depending on the type of trauma reported, discuss options including reporting the abuse to relevant authorities and/or referral for therapeutic support.

- Although it is important to assess all patients for their suitability for psychotherapeutic intervention, do not rule out the potential value of psychological interventions for patients presenting with psychotic symptoms. Depending on the resources available to you, plan how your service can develop the capacity to provide traumarelevant therapeutic interventions for patients with psychosis.

These may include mindfulness, cognitive therapy or specific trauma-focused therapies (e.g. eye-movement desensitisation reprocessing (EMDR)).

- For patients who are considered suitable for trauma-relevant psychological intervention, offer and provide this intervention as part of their treatment. Canon. responses to the experience of trauma (Longden 2012), a protective mechanism through which individuals cognitively and affectively disconnect from traumatic events that they are unable to process (Foa 1996). Given that dissociation has been associated with both trauma and AVHs in clinical and non-clinical samples, Longden and colleagues argue that the conceptualisation of AVHs as a dissociative response to stress and trauma offers a superior explanation of these phenomena (Longden 2012). To advance any psychodynamic and developmental understanding of these phenomena and their relationship with early experiences of adversity and trauma, a number of authors have recently called for the use of qualitative research designs to develop a more nuanced and refined understanding these complex human experiences (Longden 2012; Corstens 2014; Humpston 2014; McCarthy-Jones 2014; Upthegrove 2016).

\section{Clinical implications \\ Screening for early trauma}

We developed the Psychotic Symptoms Early Trauma Checklist (CAP) (Fig. 1) for use with adults who present with psychotic symptoms, but it can also be used with younger patients.

\section{Child and adolescent mental health settings}

In the child and adolescent mental health setting, determining whether or not an individual has history of trauma must remain a central feature of assessment. As a matter of course, every child presenting with any such history should also be routinely screened for psychotic experiences. Importantly, it cannot be assumed that children and adolescents who are experiencing or who have experienced psychotic phenomena will volunteer that information during an assessment. Most children and adolescents only disclose these experiences when specifically asked about them. In addition, while many of the hallucinatory and paranoid phenomena that children and adolescents report do not meet the threshold criteria for frank psychotic symptoms, their occurrence may still have clinical relevance on account of the association between such subclinical psychotic experiences and other forms of psychopathology in adolescents (Kelleher 2012). It is also important not to assume that young people with a primary presentation of depression, anxiety or personality disorder are not also experiencing some form of hallucinations or delusions.

With limited evidence to suggest that the cessation of traumatic events in the adolescent years can result in a reduction of psychotic experiences 
(Kelleher 2013a), it is important to consider the range of possible interventions that could reduce the impact of current traumas among children and adolescents presenting with psychotic symptoms. These could include family- and school-based interventions, and referral to external agencies if there is evidence of any form of abuse. Notwithstanding the concerning absence of trauma-focused therapy research for young people who report childhood trauma and psychotic experiences, therapies that address the emotional impact of any experiences of trauma, that promote self-esteem and that support the development of positive coping mechanisms also ought to be considered and offered.

\section{Adult mental health settings}

It is important to routinely assess for both childhood trauma and psychotic symptoms in individuals who present to adult mental health services. In adult services, the dominant therapeutic goal is often the management of psychotic symptoms, usually with a reliance on psychotropic medication for symptomatic control or recovery. Once symptoms have stabilised, efforts frequently then turn to occupational and functional recovery. In reality, for many adults with psychosis, little or no consideration is given to the potential origins of their psychotic symptoms, including possible experiences of childhood trauma. Given the vulnerability of some patients, clinicians can be reluctant to explore past traumas with their patients, and patients with psychosis may be denied the opportunity to talk about or explore the impact of those experiences during their engagement with mental health services.

In recent years, there has been a call for clinicians to consider the potential benefit of psychologically oriented trauma-based therapies (McCarthy-Jones 2015). These include eyemovement desensitisation and reprocessing (EMDR) (van den Berg 2015), cognitive-oriented trauma-focused therapies (McCarthy-Jones 2015) and therapies such as mindfulness (Peters 2016). There is an emerging evidence base that these therapies can be well tolerated by patients with psychosis and have therapeutic benefit. In light of these findings, there is a case for the development of targeted, evidence-based trauma-focused therapies within adult psychiatric services that are considered as a potential intervention for all psychosis patients. Expanding the suite of therapeutic interventions offered to patients with psychosis to include interventions that address a history of trauma may be a key factor in enhancing the symptomatic, emotional, social and functional trajectories of individuals for whom trauma has been a factor in the emergence of their symptoms. This would, of course, necessitate that mental health services be sufficiently resourced to recruit and/or train adequate numbers of clinical staff to provide such interventions.

\section{Conclusions}

Findings from this review suggest that: (a) there is a dynamic interplay between childhood trauma and other exposures and risk factors; (b) the role of childhood trauma needs to be considered across the continuum of psychotic outcomes and in the context of a range of psychopathological trajectories and outcomes; and (c) psychopathological outcomes associated with a history of childhood trauma are often multidimensional and do not fit within traditional diagnostic classifications.

From a clinical perspective, the complexity of the relationship between childhood trauma, psychosis and other psychopathology means that precise aetiological models for the spectrum of psychotic symptoms and disorders remain elusive. The same is true for predictive models regarding the transition from early experiences of hallucinations and delusions to later psychotic disorders. Notwithstanding these issues, screening for childhood trauma and psychotic symptoms may help in the early detection of individuals at high risk of psychotic disorder. In addition, developing and offering targeted psychotherapeutic and other interventions to reduce the potential impact of early experiences of trauma must remain an important clinical goal in reducing the incidence and subsequent impact of psychotic disorders and other psychopathology.

\section{References}

Aas M, Dazzan P, Mondelli V, et al (2014) A systematic review of cognitive function in first-episode psychosis, including a discussion on childhood trauma, stress and inflammation. Frontiers in Psychiatry, 4: 182

Abel KM, Heuvelman HP, Jörgensen L, et al (2014) Severe bereavement stress during the prenatal and childhood periods and risk of psychosis in later life: population based cohort study. BMJ, 348: $f 7679$.

Afifi TO, Mather A, Boman J, et al (2011) Childhood adversity and personality disorders: results from a nationally representative populationbased study. Journal of Psychiatric Research, 45: 814-22.

Ajnakina 0, Trotta A, Oakley-Hannibal E, et al (2016) Impact of childhood adversities on specific symptom dimensions in first-episode psychosis. Psychological Medicine, 46: 317-26.

Arseneault L, Cannon M, Fisher HL, et al (2011) Childhood trauma and children's emerging psychotic symptoms: a genetically sensitive longitudinal cohort study. American Journal of Psychiatry, 168: 65-72.

Beards S, Gayer-Anderson C, Borges S, et al (2013) Life events and psychosis: a review and meta-analysis. Schizophrenia Bulletin, 39: 740-7.

Bebbington P, Jonas S, Kuipers E, et al (2011) Childhood sexual abuse and psychosis: data from a cross-sectional national psychiatric survey in England. British Journal of Psychiatry, 199: 29-37.

Bechdolf A, Thompson A, Nelson B, et al (2010) Experience of trauma and conversion to psychosis in an ultra-high-risk (prodromal) group. Acta Psychiatrica Scandinavica, 121: 377-84. 


\section{MCQ answers}

1 a $\quad 2$ e $\quad 3$ b $\quad 4$ b $\quad 5 d$
Bentall RP, Wickham S, Shevlin M, et al (2012) Do specific early-life adversities lead to specific symptoms of psychosis? A study from the 2007 The Adult Psychiatric Morbidity Survey. Schizophrenia Bulletin, 38: $734-40$

Bentall RP, de Sousa P, Varese F, et al (2014) From adversity to psychosis: pathways and mechanisms from specific adversities to specific symptoms. Social Psychiatry and Psychiatric Epidemiology, 49: 1011-22.

Bonoldi I, Simeone E, Rocchetti M, et al (2013) Prevalence of selfreported childhood abuse in psychosis: a meta-analysis of retrospective studies. Psychiatry Research, 210: 8-15.

Bortolon C, Raffard S (2015) Self-reported psychotic-like experiences in individuals with obsessive-compulsive disorder versus schizophrenia patients: characteristics and moderation role of trait anxiety. Comprehensive Psychiatry, 57: 97-105

Catone G, Marwaha S, Kuipers E, et al (2015) Bullying victimisation and risk of psychotic phenomena: analyses of British national survey data. Lancet Psychiatry, 2: 618-24.

Clark LP (1932) Can child analysis prevent neuroses and psychoses in later life? Psychoanalytic Review, 19: 46

Corstens D, Longden E, McCarthy-Jones S, et al (2014) Emerging perspectives from the Hearing Voices Movement: implications for research and practice. Schizophrenia Bulletin, 40 (suppl 4): S285-94.

Daalman K, Diederen KMJ, Derks EM, et al (2012) Childhood trauma and auditory verbal hallucinations. Psychological Medicine, 42: 2475-84.

Duhig M, Patterson S, Connell M, et al (2015) The prevalence and correlates of childhood trauma in patients with early psychosis. Australian and New Zealand Journal of Psychiatry, 49: 651-9.

Dvir Y, Denietolis B, Frazier JA (2013) Childhood trauma and psychosis. Child \& Adolescent Psychiatric Clinics of North America, 22: 629-41.

Fisher HL, Appiah-Kusi E, Grant C (2012) Anxiety and negative selfschemas mediate the association between childhood maltreatment and paranoia. Psychiatry Research, 196: 323-4.

Fisher HL, Schreier A, Zammit S, et al (2013) Pathways between childhood victimization and psychosis-like symptoms in the ALSPAC birth cohort. Schizophrenia Bulletin, 39: 1045-55.

Foa EB, Hearst-lkeda D (1996) Emotional dissociation in response to trauma. In Handbook of Dissociation: Theoretical, Empirical, and Clinical Perspectives (eds LK Michelson, WJ Ray): 207-24. Springer USA.

Fusar-Poli P, Nelson B, Valmaggia L, et al (2014) Comorbid depressive and anxiety disorders in 509 individuals with an at-risk mental state: impact on psychopathology and transition to psychosis. Schizophrenia Bulletin, 40: 120-31.

Hayes LN, Severance EG, Leek JT, et al (2014) Inflammatory molecular signature associated with infectious agents in psychosis. Schizophrenia Bulletin, 40: 963-72.

Heim C, Nemeroff CB (2001) The role of childhood trauma in the neurobiology of mood and anxiety disorders: preclinical and clinical studies. Biological Psychiatry, 49: 1023-39.

Heim C, Newport DJ, Mletzko T, et al (2008) The link between childhood trauma and depression: insights from HPA axis studies in humans. Psychoneuroendocrinology, 33: 693-710.

Humpston CS (2014) Perplexity and meaning: toward a phenomenological "core" of psychotic experiences. Schizophrenia Bulletin, 40: 240-3.

Kelleher I, Keeley H, Corcoran P, et al (2012) Clinicopathological significance of psychotic experiences in non-psychotic young people: evidence from four population-based studies. British Journal of Psychiatry, 201: 26-32.

Kelleher I, Keeley H, Corcoran P, et al (2013a) Childhood trauma and psychosis in a prospective cohort study: cause, effect, and directionality. American Journal of Psychiatry, 170: 734-41.

Kelleher I, Murtagh A, Clarke MC, et al (2013b) Neurocognitive performance of a community-based sample of young people at putative ultra high risk for psychosis: support for the processing speed hypothesis. Cognitive Neuropsychiatry, 18: 9-25.

Kessler RC, McLaughlin KA, Greif Green J, et al (2010) Childhood adversities and adult psychopathology in the WHO World Mental Health Surveys. British Journal of Psychiatry, 197: 378-85.
Kilcommons AM, Morrison AP (2005) Relationships between trauma and psychosis: an exploration of cognitive and dissociative factors. Acta Psychiatrica Scandinavica, 112: 351-9.

Klein J, Roniger PA, Schweiger U, et al (2015) The association of childhood trauma and personality disorders with chronic depression: a cross-sectional study in depressed outpatients. Journal of Clinical Psychiatry, 76: e794-801.

Kraan T, Velthorst E, Smit F, et al (2015) Trauma and recent life events in individuals at ultra high risk for psychosis: review and meta-analysis. Schizophrenia Research, 161: 143-9.

Longden E, Madill A, Waterman MG (2012) Dissociation, trauma, and the role of lived experience: toward a new conceptualization of voice hearing. Psychological Bulletin, 138: 28-76.

Longden E, Sampson M, Read J (2016) Childhood adversity and psychosis: generalised or specific effects? Epidemiology and Psychiatric Sciences, 25: $349-59$.

MacDonald EM, Hayes RL, Baglioni Jr AJ (2000) The quantity and quality of the social networks of young people with early psychosis compared with closely matched controls. Schizophrenia Research, 46: $25-30$

MacDougall AA (1939) Psychological casualties in war. BMJ, 2: 1203.

Martindale BV (2007) Psychodynamic contributions to early intervention in psychosis. Advances in Psychiatric Treatment, 13: 34-42.

Martindale B, Summers A (2013) The psychodynamics of psychosis. Advances in Psychiatric Treatment, 19: 124-31.

Matheson SL, Shepherd AM, Pinchbeck RM, et al (2013) Childhood adversity in schizophrenia: a systematic meta-analysis. Psychological Medicine, 43: 225-38.

McCarthy-Jones S, Trauer T, Mackinnon A, et al (2014) A new phenomenological survey of auditory hallucinations: evidence for subtypes and implications for theory and practice. Schizophrenia Bulletin, 40: $231-5$

McCarthy-Jones S, Longden E (2015) Auditory verbal hallucinations in schizophrenia and post-traumatic stress disorder: common phenomenology, common cause, common interventions? Frontiers in Psychology, 6: 1071

McEwen BS (2000) Allostasis and allostatic load: implications for neuropsychopharmacology. Neuropsychopharmacology, 22: 108-24.

Millan MJ, Andrieux A, Bartzokis G, et al (2016) Altering the course of schizophrenia: progress and perspectives. Nature Reviews Drug Discovery, 15: 485-515.

Morgan C, Reininghaus U, Reichenberg A, et al (2014) Adversity, cannabis use and psychotic experiences: evidence of cumulative and synergistic effects. British Journal of Psychiatry, 204: 346-53.

Muenzenmaier KH, Seixas AA, Schneeberger AR, et al (2015) Cumulative effects of stressful childhood experiences on delusions and hallucinations. Journal of Trauma and Dissociation, 16: 442-62.

Mueser KT, Rosenberg SD, Goodman LA, et al (2002) Trauma, PTSD, and the course of severe mental illness: an interactive model. Schizophrenia Research, 53: 123-43.

Peters E, Ward T, Jackson M, et al (2016) Clinical, socio-demographic and psychological characteristics in individuals with persistent psychotic experiences with and without a "need for care". World Psychiatry, 15. $41-52$.

Read J, van Os J, Morrison AP, et al (2005) Childhood trauma, psychosis and schizophrenia: a literature review with theoretical and clinical implications. Acta Psychiatrica Scandinavica, 112: 330-50.

Reiff M, Castille DM, Muenzenmaier K, et al (2012) Childhood abuse and the content of adult psychotic symptoms. Psychological Trauma: Theory, Research, Practice, and Policy, 4: 356-69.

Schroeder K, Fisher HL, Schäfer I (2013) Psychotic symptoms in patients with borderline personality disorder: prevalence and clinical management. Current Opinion in Psychiatry, 26: 113-9.

Seo D, Tsou KA, Ansell EB, et al (2014) Cumulative adversity sensitizes neural response to acute stress: association with health symptoms. Neuropsychopharmacology, 39: 670-80. 
Sheffield JM, Williams LE, Blackford JU, et al (2013) Childhood sexual abuse increases risk of auditory hallucinations in psychotic disorders. Comprehensive Psychiatry, 54: 1098-104.

Shevlin M, Murphy J, Read J, et al (2011a) Childhood adversity and hallucinations: a community-based study using the National Comorbidity Survey Replication. Social Psychiatry and Psychiatric Epidemiology, 46: 1203-10.

Shevlin M, Armour C, Murphy J, et al (2011b) Evidence for a psychotic posttraumatic stress disorder subtype based on the National Comorbidity Survey. Social Psychiatry and Psychiatric Epidemiology, 46: 1069-78.

Sitko K, Bentall RP, Shevlin M, et al (2014) Associations between specific psychotic symptoms and specific childhood adversities are mediated by attachment styles: an analysis of the National Comorbidity Survey. Psychiatry Research, 217: 202-9.

Thompson AD, Nelson B, Pan Yuen H, et al (2014) Sexual trauma increases the risk of developing psychosis in an ultra high-risk "prodromal" population. Schizophrenia Bulletin, 40: 697-706.

Trotta A, Murray RM, Fisher HL (2015) The impact of childhood adversity on the persistence of psychotic symptoms: a systematic review and metaanalysis. Psychological Medicine, 45: 2481-98.

Upthegrove R, Chard C, Jones L, et al (2015) Adverse childhood events and psychosis in bipolar affective disorder. British Journal of Psychiatry, 206: 191-7.

Upthegrove R, Broome MR, Caldwell K, et al (2016) Understanding auditory verbal hallucinations: a systematic review of current evidence. Acta Psychiatrica Scandinavica, 133: 352-67.

Valmaggia LR, Day FL, Kroll J, et al (2015) Bullying victimisation and paranoid ideation in people at ultra high risk for psychosis. Schizophrenia Research, 168: 68-73.

van Dam DS, van der Ven E, Velthorst E, et al (2012) Childhood bullying and the association with psychosis in non-clinical and clinical samples: a review and meta-analysis. Psychological Medicine, 42: 2463-74. van den Berg DP, de Bont PA, van der Vleugel BM, et al (2015) Prolonged exposure vs eye movement desensitization and reprocessing vs waiting list for posttraumatic stress disorder in patients with a psychotic disorder: a randomized clinical trial. JAMA Psychiatry, 72: 259-67.

van Nierop M, Lataster T, Smeets F, et al (2014) Psychopathological mechanisms linking childhood traumatic experiences to risk of psychotic symptoms: analysis of a large, representative population-based sample. Schizophrenia Bulletin, 40 (suppl 2): S123-30.

van Os J, Rutten BBF, Poulton R (2008) Gene-environment interactions in schizophrenia: review of epidemiological findings and future directions. Schizophrenia Bulletin, 34: 1066-82.

van Os J, Linscott RJ, Myin-Germeys I, et al (2009) A systematic review and meta-analysis of the psychosis continuum: evidence for a psychosis proneness-persistence-impairment model of psychotic disorder. Psychological Medicine, 39: 179-95.

van Os J, Kenis G, Rutten BPF (2010) The environment and schizophrenia. Nature, 468: 203-12.

Varese F, Smeets F, Drukker M, et al (2012) Childhood adversities increase the risk of psychosis: a meta-analysis of patient-control, prospectiveand cross-sectional cohort studies. Schizophrenia Bulletin, 38: 661-71.

Vassos E, Pedersen CB, Murray RM, et al (2012) Meta-analysis of the association of urbanicity with schizophrenia. Schizophrenia Bulletin, 38 : 1118-23.

Velikonja T, Fisher HL, Mason 0, et al (2015) Childhood trauma and schizotypy: a systematic literature review. Psychological Medicine, 45: 947-63.

Velthorst E, Nelson B, O'Connor K, et al (2013) History of trauma and the association with baseline symptoms in an ultra-high risk for psychosis cohort. Psychiatry Research, 210: 75-81.

Wolke D, Lereya ST, Fisher HL, et al (2014) Bullying in elementary school and psychotic experiences at 18 years: a longitudinal, population-based cohort study. Psychological Medicine, 44: 2199-211.

\section{MCOs}

Select the single best option for each question stem

1 Which of the following best describes the association between childhood trauma and psychosis reported in recent meta-analyses?

a Childhood trauma is associated with both nonclinical psychotic symptoms and pathological expressions of psychosis

b When compared with people with depression, individuals with a diagnosis of schizophrenia are significantly more likely to have a history of childhood trauma

c There is no difference in the strength of the association between childhood trauma and psychotic outcomes when you compare population-based samples with clinical samples

$d$ There is no evidence that the experience of more than one trauma is associated with an increased risk of psychosis

e Childhood trauma is only associated with pathological psychotic outcomes.

2 Given that there is a known relationship between childhood trauma and psychosis, which of the following statements is the most accurate?

a The association between childhood trauma and psychosis is only present among those with a pre-existing genetic vulnerability b The association between childhood trauma and psychosis is only activated when people are exposed to additional risk factors, such as cannabis use and social adversity

c It is only when a pre-existing genetic vulnerability, childhood trauma and exposure to additional risk factors interact that there is a risk of psychosis

$\mathrm{d}$ The combination of pre-existing genetic vulnerability, the experience of childhood trauma and exposure to additional risk factors is only associated with psychotic disorder

e None of the above.

3 Which of the following childhood traumas has been found to be associated with transition from a high-risk state to diagnosable psychotic disorder?

a Emotional abuse

b Sexual abuse

c Physical abuse

d Parental death

e All of the above.

4 A 17-year-old adolescent presents with a history of low mood and anxiety. The onset of his depression and anxiety followed an extended period of bullying as a child. He does not report any additional childhood trauma. You screen him for psychotic symptoms and he reports having heard voices since he was a child. What is the most likely clinical relevance of this?

a This should be considered as an unusual clinical presentation and there is a high likelihood that he will develop more pathological psychotic outcomes in adulthood

b A clinical presentation with concurrent depression, anxiety and auditory hallucinations can be expected and it is important to explore all of these symptoms over the course of his treatment

c His auditory hallucinations have no clinical relevance as his primary difficulties relate to his mood and anxiety

d He should be commenced on a psychotropic immediately

e None of the above.

5 Which of the following has been found to have the strongest association with paranoia specifically?

a Childhood sexual abuse

b Parental death

c Childhood neglect

d Institutional care

e Urban living 